\title{
Endovascular recanalization of chronic total occlusions of the native superficial femoral artery after failed femoropopliteal bypass in patients with critical limb ischemia
}

Roberto Minici ${ }^{1 *}$ (D) Michele Ammendola ${ }^{2}$, Marisa Talarico ${ }^{3}$, Maria Luposella $^{4}$, Marco Minici $^{5}$, Salvatore Ciranni ${ }^{6}$, Giuseppe Guzzardi ${ }^{7}$ and Domenico Laganà ${ }^{1}$

\begin{abstract}
Background: Femoropopliteal bypass occlusions are a significant issue in patients with critical limb ischemia and chronic total occlusion of the native superficial femoral artery, which challenges vascular surgeons and interventional radiologists. Performing a secondary femoropopliteal bypass is still considered the standard of care, although it is associated with a higher complication rate and lower patency rate in comparison with primary bypass. Over the past few years, angioplasty has been commonly used, with the development in endovascular technologies, to treat chronic total occlusions of the native superficial femoral artery, with a good technical success rate and clinical prognosis. The purpose of the study is to assess the outcome of endovascular recanalization of chronic total occlusions of the native superficial femoral artery, in patients unfit for surgery with critical limb ischemia after failed femoropopliteal bypass.
\end{abstract}

Results: A total of 54 patients were treated. $77.8 \%$ of the conduits were PTFE grafts; the remainder were singlesegment great saphenous veins. The most common clinical presentation was rest pain. Technical success was achieved in 51 (94.4\%) of 54 limbs. Angiographically, $77.8 \%$ of the lesions were TASC II category D, while $22.2 \%$ were TASC II category C. The average length of the native SFA lesions was $26.8 \mathrm{~cm}$. Clinical success, with improved Rutherford classification staging, followed each case of technical success. The median follow-up value was 5.75 years (IQR, 1.5-7). By Kaplan-Meier survival analysis, primary patency rates were $61 \%$ ( $\pm 0.07 \mathrm{SE}$ ) at 1 year and $46 \%$ $( \pm 0.07 \mathrm{SE})$ at 5 years. Secondary patency rates were $93 \%( \pm 0.04 \mathrm{SE})$ at 1 year and $61 \%( \pm 0.07 \mathrm{SE})$ at 5 years. Limb salvage rates were $94 \%( \pm 0.03 \mathrm{SE})$ at 1 year and $88 \%( \pm 0.05 \mathrm{SE})$ at 5 years.

Conclusions: The endovascular recanalization of chronic total occlusions (CTO) of the native superficial femoral artery (SFA) after a failed femoropopliteal bypass is a safe and effective therapeutic option in patients unfit for surgery with critical limb ischemia.

Keywords: Femoropopliteal bypass occlusion, Native SFA recanalization, Chronic total occlusion, Endovascular recanalization, Critical limb ischemia

\footnotetext{
* Correspondence: robertominici@tiscali.it

'Radiology Division, Department of Experimental and Clinical Medicine, Magna Graecia University of Catanzaro, University Hospital Mater Domini, Viale Europa, 88100 Catanzaro, Italy

Full list of author information is available at the end of the article
}

\section{Springer Open}

(-) The Author(s). 2021 Open Access This article is licensed under a Creative Commons Attribution 4.0 International License, which permits use, sharing, adaptation, distribution and reproduction in any medium or format, as long as you give appropriate credit to the original author(s) and the source, provide a link to the Creative Commons licence, and indicate if changes were made. The images or other third party material in this article are included in the article's Creative Commons licence, unless indicated otherwise in a credit line to the material. If material is not included in the article's Creative Commons licence and your intended use is not permitted by statutory regulation or exceeds the permitted use, you will need to obtain permission directly from the copyright holder. To view a copy of this licence, visit http://creativecommons.org/licenses/by/4.0/. 


\section{Background}

According to the Trans Atlantic Inter-Society Consensus for the Management of Peripheral Arterial Disease II (TASC II), surgical revascularization with bypass grafting is the treatment of choice for chronic total occlusions (CTO) of the superficial femoral artery (SFA), with favourable technical success rate and clinical prognosis (Norgren et al. 2007). However, the occlusion of lower limb arterial bypass remains a significant problem, which challenges vascular surgeons and interventional radiologists. The graft occlusion rate was estimated to be 5$10 \%$ in the early period after surgery ( $<30$ days) (Ziegler et al. 2011; Owens et al. 2008a, b; Brewster et al. 1983) and $20-50 \%$ in the late period (> 30 days) (Donaldson et al. 1991; Leather et al. 1988; Taylor et al. 1990). Furthermore, in patients with bypass graft failure, the average limb salvage rate is only about $50 \%$ at 2 years (Taylor et al. 1990).

After a bypass graft failure has occurred, the possible targets of intervention are the bypass graft itself or the native SFA. Among interventions on bypass graft, surgical or endovascular approaches can be distinguished. Performing a secondary femoropopliteal bypass is still considered the standard of care, although it is associated with a higher complication rate (perioperative morbidity of $25 \%$ ) and lower mid-term patency rate in comparison with primary bypass (Baldwin et al. 2004; Belkin et al. 1995). Advanced age, lack of a good great saphenous vein, anastomosis' pseudoaneurysms and high surgical risks make the surgical approach not always suitable. Options to achieve endovascular recanalization of bypass graft include thrombectomy and catheter-directed thrombolysis (CDT), but both of them correlates with poor long-term limb salvage and patency rates (Domínguez Paillacho et al. 2018; Kalinowski et al. 2003; Nehler et al. 2003). In addition to the above, contraindications to lysis should be considered.

Over the past few years, angioplasty has been commonly used, with the development in endovascular technologies, to treat chronic total occlusions of the native SFA, with a good technical success rate and clinical prognosis (Mewissen et al. 2004). Hence, the idea to recanalize the native SFA chronic total occlusions has been born in patients with critical limb ischemia (CLI) and femoro-popliteal bypass failure, limited to those cases unfit for surgery or refusing surgical reconstruction. Data regarding this approach in femoro-popliteal bypass failure are limited to few case-series studies ( $\mathrm{Li}$ et al. 2018; Gandini et al. 2009; Davies et al. 2017), so the need for new studies should be emphasized to better understand long-term outcomes of this option compared to secondary bypass surgery.

This study aims to assess the outcome of endovascular recanalization of chronic total occlusions (CTO) of the native superficial femoral artery (SFA), in patients unfit for surgery with critical limb ischemia after failed femoropopliteal bypass.

\section{Methods \\ Study design}

The Institutional Review Board approval and informed written consent from each patient have been obtained. This study is a single-centre, retrospective analysis of prospectively collected data of consecutive patients with CLI, who had undergone, from January 2013 to December 2020, endovascular recanalization of CTOs of the native SFA after the failure of a femoropopliteal bypass. Inclusion criteria, met by all patients, are (I) occlusion of a previous femoropopliteal bypass graft with CTO of the native SFA; (II) duration of ischemia symptoms $>14$ days; (III) evaluation by a multidisciplinary team of vascular surgeons, interventional radiologists and anaesthetists; (IV) refusal of surgical reconstruction by patients or being considered unfit for surgery: absence of an adequate great saphenous vein, poor distal bypass target vessels, severe comorbidities (acute coronary syndrome or stroke within the previous 6 weeks, severe chronic obstructive pulmonary disease). Exclusion criteria are: (I) glomerular filtration rate $(\mathrm{GFR})<30 \mathrm{~mL} / \mathrm{min}$ in nondialyzing patients; (II) previous end-to-end anastomosis; (III) previous endarterectomy or endovascular procedures at the treatment site; (IV) untreated ipsilateral iliac stenosis $>70 \%$; (V) contraindications to heparin or antiplatelet drugs. In all cases, a computed tomography $(\mathrm{CT})$ angiography was performed to plan the recanalization procedure; the ankle-brachial index (ABI) and the symptomatic classification by Rutherford were recorded before and after endovascular treatment. Catheter-directed thrombolysis (CDT), available for patients presenting with acute limb ischemia (ALI), was not offered to patients as a treatment option considering the duration of ischemia symptoms $>14$ days among inclusion criteria.

\section{Treatment}

The endovascular procedure was performed in dedicated angiographic suites. After local anaesthesia, in most cases, a retrograde approach by puncture of the contralateral common femoral artery was used. Ultrasoundguided cannulation of the common femoral artery with the Seldinger technique was performed and a $6 \mathrm{Fr}$ vascular sheath (Radifocus ${ }^{\circ}$, Terumo) was positioned. In the case of iliac arteries obstruction, radial or humeral arterial access was realized, with a 5Fr vascular sheath (Radifocus ${ }^{\circ}$ II, Terumo) used in cases of transradial approach. When it has been possible, a dual anti-platelet therapy (in most cases, acetylsalicylic acid $100 \mathrm{mg} /$ day plus clopidogrel $75 \mathrm{mg} /$ day) has been started 3 days before endovascular treatment, and, in all cases, it has been 
administered at least for 1 year after endovascular treatment. Heparin sodium (100 IU/Kg) has been administered in the arterial lumen systematically, to maintain an activated clotting time $>250$ s. Lesions of native SFA, mainly calcific, were generally crossed with 0.035-in. hydrophilic guidewire (Terumo), eventually using subintimal/SAFARI/retrograde popliteal puncture techniques and Navicross ${ }^{\oplus}$ (Terumo) support catheters. PTA was performed using low-profile .035" balloons (Ultraverse ${ }^{\oplus}$, Bard), with $180 \mathrm{~s}$ average inflation times and various lengths and dimensions chosen by the operator. Stenting was performed only in the cases of PTA failure, defined by severe elastic recoil or flow-limiting dissection or residual stenosis $>30 \%$, using vascular self-expanding nitinol stents (E-Luminexx ${ }^{\mathrm{T \mu}}$, Bard). Eventually, infrapopliteal arteries were treated simultaneously to ensure at least one patent BTK vessel. Vascular closure devices (Angio-Seal ${ }^{\text {TM }}$ VIP, Terumo) and radial artery compression devices (TR Band ${ }^{\oplus}$, Terumo) were used according to instructions for use; in the other cases, vascular access site hemostasis was achieved by manual compression (Figs. 1 and 2).

\section{Definitions}

According to the Society for Vascular Surgery reporting standards, technical success was defined by residual stenosis less than $30 \%$, with the absence of stasis of contrast medium and restoration of direct flow in treated segments. Clinical success was defined by an improvement on the Rutherford classification staging, therefore by an improved pain-free walking distance, resting pain resolution or ischemic ulcer healing. Reporting standards of the SVS were used to define primary, assisted-primary and secondary patency rates, too (Rutherford et al. 1997; Ahn et al. 1993).

\section{Follow-up}

Patients underwent routine follow-up with clinical examination, ABI evaluation and duplex ultrasound imaging, scheduled at 1,3 , and 6 months after the procedure and every 6 months thereafter. CT-angiography was performed only in case of recurrent symptoms, any drop in ABIs of 0.15 or more, or peak systolic velocity ratio > 2.5 on duplex-US.

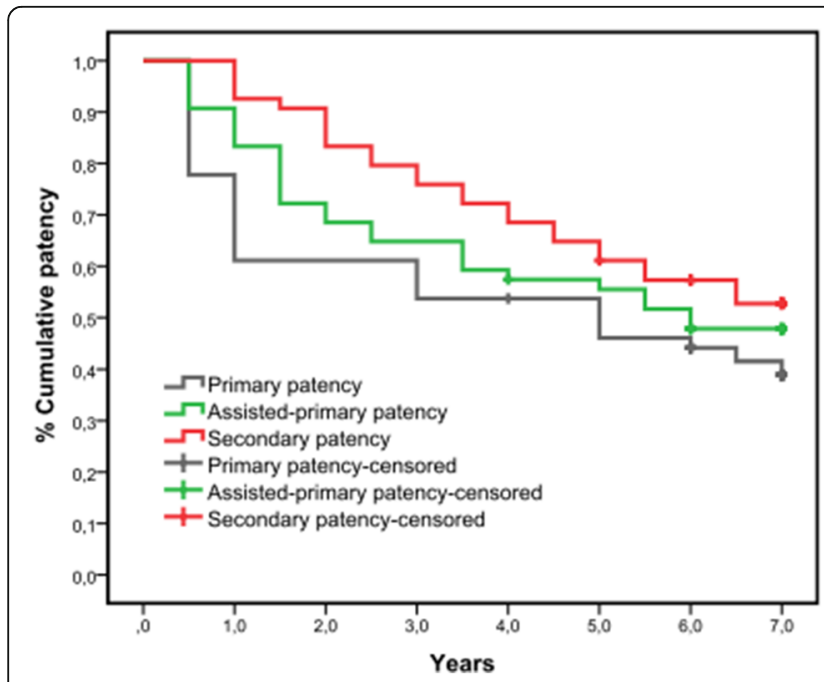

Cumulative Rates

- Primary patency

Assisted-primary patency

Secondary patency

Amputation-free survival

Limb salvage
At 1 year

rate $( \pm \mathrm{SE})$ - numbers at risk

$61 \%( \pm 0.07)-42$

$83 \%( \pm 0.05)-49$

$93 \%( \pm 0.04)-50$

$93 \%( \pm 0.04)-50$

$94 \%( \pm 0.03)-51$

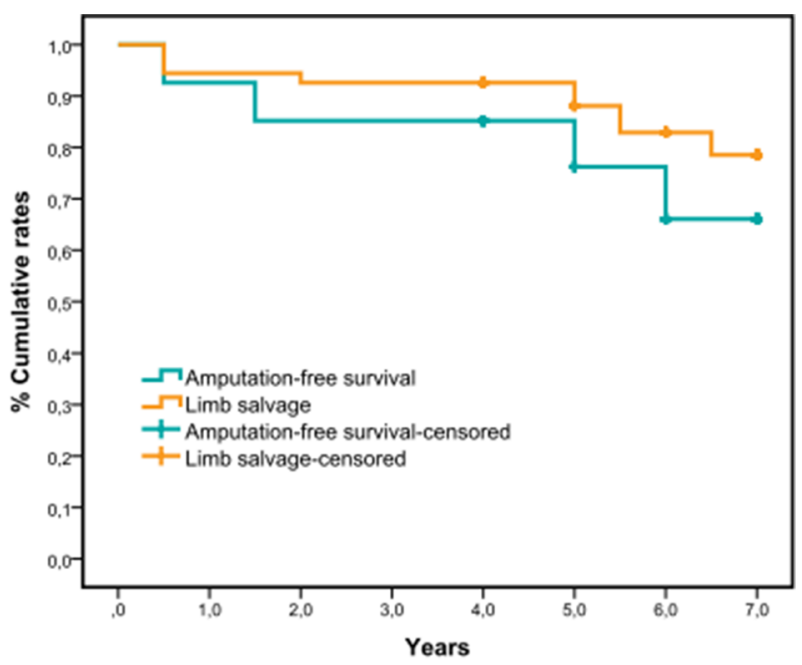

At 3 years

rate $( \pm \mathrm{SE})$ - numbers at risk

At 5 years

$54 \%( \pm 0.07)-33$

rate $( \pm \mathrm{SE})$ - numbers at risk

$46 \%( \pm 0.07)-27$

$65 \%( \pm 0.06)-36$

$55 \%( \pm 0.07)-30$

$76 \%( \pm 0.06)-43$

$61 \%( \pm 0.07)-35$

$85 \%( \pm 0.05)-46$

$76 \%( \pm 0.06)-38$

$93 \%( \pm 0.04)-41$

$88 \%( \pm 0.05)-41$

Fig. 13 D reconstruction of a computed tomography angiography (A) showing the failure of the femoropopliteal bypass graft (arrows), whose target is the below-knee popliteal artery, and complete occlusion of the left superficial femoral artery, immediately after its origin, confirmed by a digital subtraction angiography 


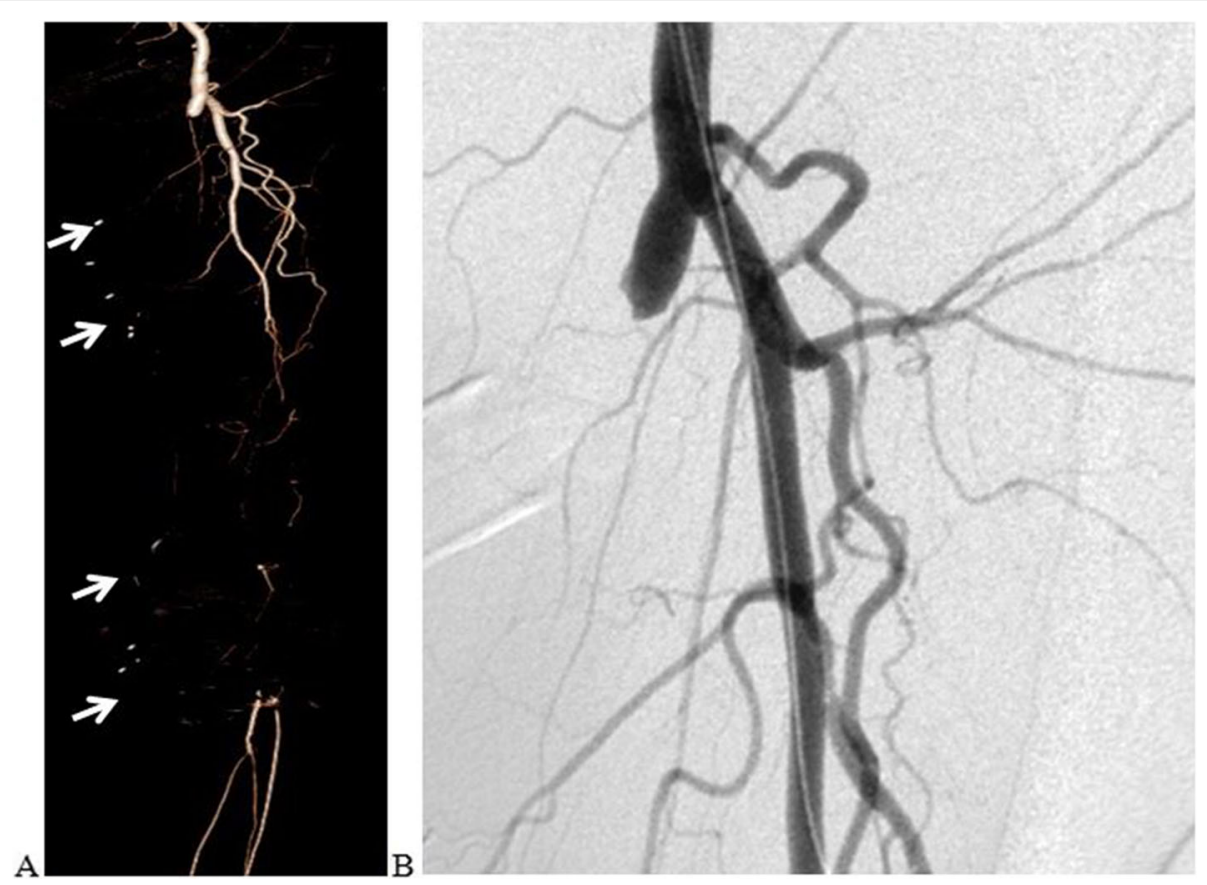

Fig. 2 Fluoroscopic images (A) demonstrating percutaneous transluminal angioplasty of all portions of the left superficial femoral artery. Subsequent angiography (B) shows PTA failure, defined by a flow-limiting dissection, after which multiple stenting is performed (C). Digital subtraction angiography (D) demonstrates successful recanalization of the left superficial femoral artery. A computed tomography angiography with 3D reconstruction (E), performed at 12 months follow-up, confirms patency of the native left superficial femoral artery

\section{Statistical analysis}

Data were maintained in an Excel spreadsheet (Microsoft Inc, Redmond, Wash) and the statistical analyses were performed on an intention-to-treat basis, using SPSS software (SPSS, version 22 for Windows; SPSS Inc, Chicago IL, USA). Kolmogorov-Smirnov test and Shapiro-Wilk test were used to verify the normality assumption of data. Categorical data are presented as frequency (percentage value). Continuous normally distributed data are presented as mean \pm standard deviation. Continuous not normally distributed data are presented as median (interquartile range: 25th and 75th percentiles - IQR). The unpaired Student t-test was used to assess statistical differences for continuous normally distributed data, while categorical and continuous not normally distributed data were assessed using the Chisquared test and the Mann-Whitney test, respectively. Kaplan-Meier survival analysis was performed to assess time-dependent outcomes, and comparisons were made with the log-rank test. The independence between censored data and the tested event was assessed by clinical evaluation and telephone contacts in the cases of withdrawal. Hence, the assumption of independent censoring was met, avoiding bias regarding the observed timedependent data. Univariate and multivariate analyses, using Cox proportional hazards regression models, were performed to identify patient/lesion characteristics associated with primary patency loss. A P-value of $<0.05$ was considered statistically significant for the aforementioned tests.

\section{Results}

During the study interval (January 2013 - December 2020), a total of 54 patients (59.2\% male; average age 71.9 years) underwent endovascular recanalization of CTOs of the native SFA, after failed femoropopliteal bypass graft and clinical presentation with CLI (duration of ischemia symptoms $>14$ days). The aforementioned patients have refused surgical reconstruction or have been considered unfit for surgery by a multidisciplinary team. $33.3 \%$ of the failed femoropopliteal bypass graft were to the above-knee popliteal artery, while the remaining $66.7 \%$ were to the below-knee popliteal artery. A total of $77.8 \%$ of the conduits were PTFE grafts and the rest were single-segment great saphenous veins. The most common clinical presentation was the rest pain (category IV according to the Rutherford classification for CLI), observed in $55.5 \%$ of the patients. Demographics and comorbidities for the study population are reported in Table 1.

The median patency of the previous femoropopliteal bypass graft was 29 months. Angiographically, $77.8 \%$ of the lesions were TASC II category D, while $22.2 \%$ TASC II category $C$. The average length of the native SFA 
Table 1 Population data

\begin{tabular}{ll}
\hline Variables & All patients $(\boldsymbol{n}=\mathbf{5 4})$ \\
\hline Age (years) & $71.9( \pm 12.8)$ \\
Male & $32(59.2 \%)$ \\
BMI & $23.8(17.2-29.9)$ \\
Pre-procedure ABI & $0.3(0.1-0.5)$ \\
Risk factors & \\
Diabetes mellitus & $27(50 \%)$ \\
Coronary artery disease & $35(64.8 \%)$ \\
Congestive heart failure & $13(24.1 \%)$ \\
Cerebrovascular disease & $9(16.7 \%)$ \\
Smoking history & $45(83.3 \%)$ \\
Current smoker & $5(9.3 \%)$ \\
Hypertension & $51(94.4 \%)$ \\
Hyperlipidaemia & $48(88.9 \%)$ \\
Chronic renal insufficiency(eGFR $<90$ mL/min) & $10(18.5 \%)$ \\
Rutherford categories at presentation & \\
1 or 2 or 3 - claudication & $19(35.2 \%)$ \\
4 - rest pain & $30(55.5 \%)$ \\
5 or 6 - tissue loss & $5(9.3 \%)$ \\
Bypass graft type & \\
Great saphenous vein & $12(22.2 \%)$ \\
PTFE & $42(77.8 \%)$ \\
Bypass graft target & \\
Above-knee popliteal artery & \\
Below-knee popliteal artery & \\
\hline & \\
&
\end{tabular}

lesions was $26.8 \mathrm{~cm}$. The below-the-knee (BTK) runoff, described as the number of patent vessels among anterior tibial, posterior tibial and peroneal arteries, was 2 vessels in $50 \%$ of the patients. $74.1 \%$ of the patients had undergone a contralateral femoral plus ipsilateral retrograde popliteal vascular access, $22.2 \%$ a contralateral femoral vascular access and $3.7 \%$ a radial or humeral vascular access. Among recanalization techniques, subintimal angioplasty was performed in 38 patients (70.4\%), while intraluminal and SAFARI techniques were both performed in 8 patients $(14.8 \%)$ respectively. A total of 48 patients $(88.9 \%)$ underwent stenting, due to the PTA failure, with a median value of 2 stents positioned per patient. Technical success was achieved in 51 (94.4\%) of 54 limbs. Three technical failures occurred, two caused by unsuccessful recanalization despite attempts with the SAFARI technique and one caused by severe impassable fibrosis of the distal bypass anastomosis. In one case after successful recanalization, a distal thromboembolic occlusion occurred, immediately resolved by catheter thrombus aspiration with a 6-Fr guiding catheter. No other vascular complications occurred during the procedures. The mean duration of all interventions was 119
$( \pm 39)$ minutes with a mean fluoroscopy time of $38( \pm$ 21) minutes. Lesion and procedure data are detailed in Table 2.

Clinical success, with improved Rutherford classification staging, followed each case of technical success. The Mann-Whitney test was used to identify a statistically significant $(P=0.04)$ difference between pre-procedure (0.3; IQR: $0.1-0.5)$ and post-procedure (0.6; IQR: $0.4-$ $0.8)$ ABI. The median follow-up value was 5.75 years (IQR, 1.5-7). By Kaplan-Meier survival analysis, primary patency rates were $61 \%( \pm 0.07 \mathrm{SE})$ at 1 year, $54 \%( \pm$ $0.07 \mathrm{SE})$ at 3 years and $46 \%( \pm 0.07 \mathrm{SE})$ at 5 years. Assisted-primary patency rates were $83 \%( \pm 0.05 \mathrm{SE})$ at 1 year, $65 \%( \pm 0.06 \mathrm{SE})$ at 3 years and $55 \%( \pm 0.07 \mathrm{SE})$ at 5 years. Secondary patency rates were $93 \%( \pm 0.04$ $\mathrm{SE})$ at 1 year, $76 \%( \pm 0.06 \mathrm{SE})$ at 3 years and $61 \%( \pm$ $0.07 \mathrm{SE}$ ) at 5 years. At follow-up, 14 patients presented with acute stent thrombosis and were treated with thrombectomy or catheter-directed thrombolysis (CDT) plus angioplasty; in 4 cases amputation at metatarsal level was necessary. Out of the patients presenting at

Table 2 Lesion and procedure data

\begin{tabular}{ll}
\hline Variables & $\begin{array}{l}\text { All patients }(\boldsymbol{n}= \\
\mathbf{5 4} \mathbf{L}\end{array}$ \\
\hline Patency of previous bypass graft (months) & $29(6-76)$ \\
Lesion length (cm) & $26.8( \pm 4.8)$ \\
TASC II category & \\
TASC C & $12(22.2 \%)$ \\
TASC D & $42(77.8 \%)$ \\
BTK runoff & \\
3 vessels & $8(14.8 \%)$ \\
2 vessels & $27(50 \%)$ \\
1 vessel & $19(35.2 \%)$ \\
Vascular access-site & \\
Contralateral Femoral + ipsilateralretrograde & $40(74.1 \%)$ \\
popliteal & \\
Contralateral Femoral & $12(22.2 \%)$ \\
Radial or Humeral & $2(3.7 \%)$ \\
Recanalization technique & \\
Intraluminal & $8(14.8 \%)$ \\
Subintimal & $38(70.4 \%)$ \\
SAFARI & $8(14.8 \%)$ \\
Technical success & $51(94.4 \%)$ \\
Patients receiving stent placement & $48(88.9 \%)$ \\
Stents positioned per patient & $2(1-5)$ \\
Procedure duration (min) & $119( \pm 39)$ \\
Fluoroscopy time (min) & $38( \pm 21)$ \\
Fost-procedure ABI & $0.6(0.4-0.8)$ \\
\hline & $5.75(1.5-7)$. \\
\hline
\end{tabular}


follow-up with chronic CLI symptoms (20/54), nineteen underwent endovascular reintervention and in 5 cases an above-knee amputation was performed. During the follow-up period, five patients died of other causes, four of which were from myocardial infarction, and two patients missed routine follow-up, consequently considered as natural dropouts as well as censored event-unrelated cases after a telephone interview. Amputation-free survival rates were $93 \%( \pm 0.04 \mathrm{SE})$ at 1 year, $85 \%( \pm 0.05$ $\mathrm{SE})$ at 3 years and $76 \%( \pm 0.06 \mathrm{SE})$ at 5 years. Limb salvage rates were $94 \%( \pm 0.03 \mathrm{SE})$ at 1 year, $93 \%( \pm 0.04$ $\mathrm{SE})$ at 3 years and $88 \%( \pm 0.05 \mathrm{SE})$ at 5 years. Timedependent outcomes assessed by Kaplan-Meier survival analysis are detailed in Fig. 3.

Cox proportional hazards regression models were used to determine predictors of primary patency loss for the entire cohort of patients (Table 3). By univariate analysis, statistically significant predictors of primary patency loss were diabetes mellitus (HR 4.2; $P=0.03$ ), chronic renal insufficiency (eGFR $<90 \mathrm{~mL} / \mathrm{min}$ ) (HR 3.9; $P=0.04$ ), tissue loss at clinical presentation (HR 4.1; $P=0.04$ ), bypass target to below-knee popliteal artery (HR 4.3; $P=0.03$ ), lesion length $\geq 30 \mathrm{~cm}$ (HR 4.5; $P=0.02$ ), TASCII D lesion (HR 4.2; $P=0.03$ ), BTK runoff (1 vessel) (HR 5.5; $P=0.01$ ) and stents positioned per patient $\geq 3$ (HR 3.9; $P=0.04$ ). Age, age $\geq 70$ years, sex (male), bmi, coronary artery disease, congestive heart failure, cerebrovascular disease, smoking history, current smoker, hypertension, hyperlipidaemia, claudication or rest pain at clinical presentation were not predictors of primary patency loss. By multivariate analysis, statistically significant predictors of primary patency loss were diabetes mellitus (HR 6.1; $P=0.04$ ), chronic renal insufficiency (eGFR $<90 \mathrm{~mL} /$ min) (HR 4.6; $P=0.04)$, bypass target to below-knee popliteal artery (HR 4.1; $P=0.04$ ), lesion length $\geq 30 \mathrm{~cm}$ (HR 4.6; $P=0.04$ ), TASCII D lesion (HR 5.7; $P=0.04$ )
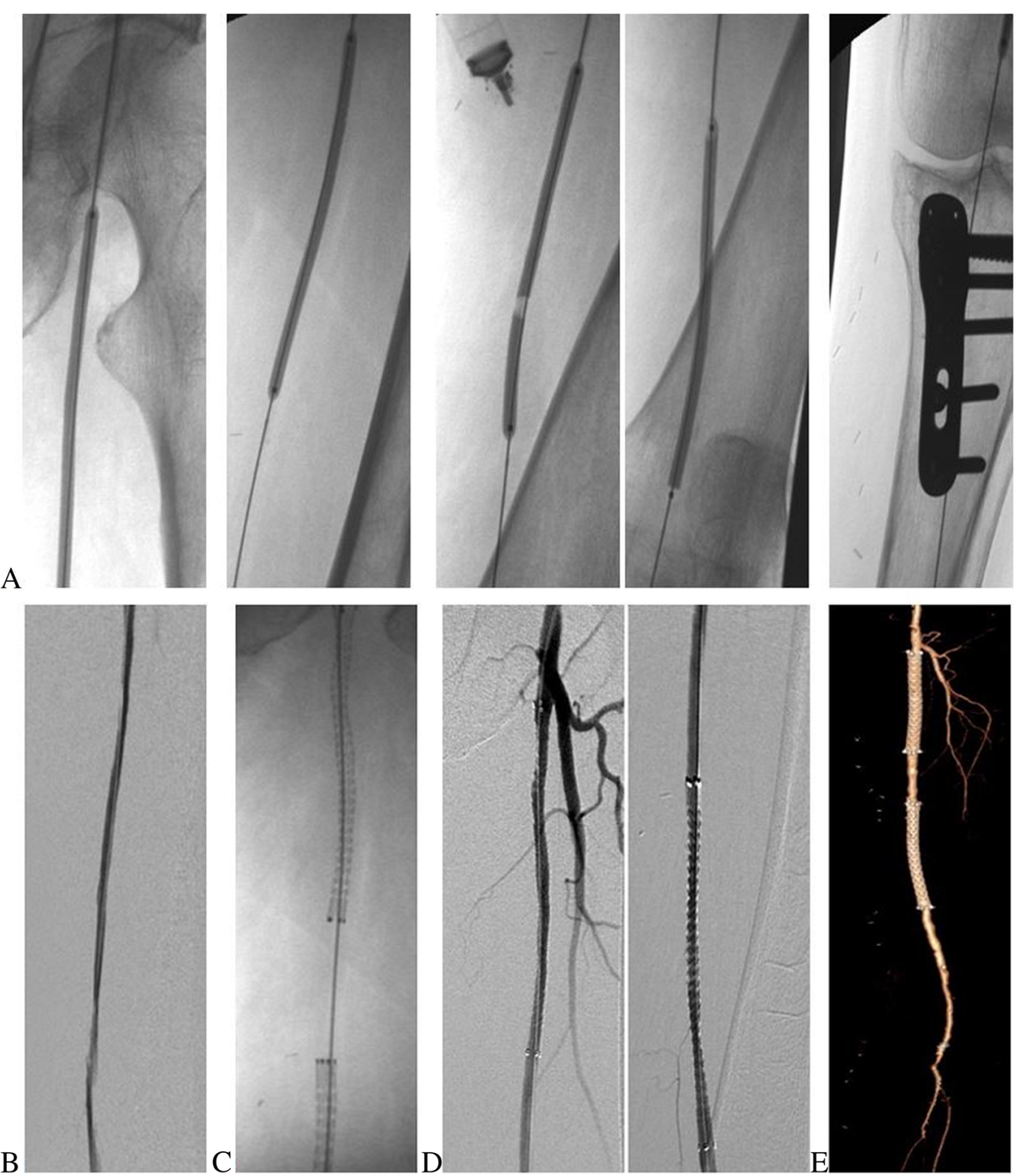

Fig. 3 Time-dependent outcomes assessed by Kaplan-Meier survival analysis 
Table 3 Predictors of primary patency loss by Cox proportional hazards regression models

\begin{tabular}{|c|c|c|}
\hline Variable & UnivariateHR $(95 \% \mathrm{Cl})-P$ value & MultivariateHR $(95 \% \mathrm{Cl})-P$ value \\
\hline Age & $1.2(0.9-1.3)-0.59$ & $1.3(0.8-2.2)-0.71$ \\
\hline Age $\geq 70$ years & $1.4(1.0-1.5)-0.53$ & $1.5(0.6-9.7)-0.65$ \\
\hline Sex (Male) & $0.9(0.7-1.3)-0.81$ & $2.1(0.5-13.4)-0.88$ \\
\hline BMI & $1.1(0.9-1.5)-0.79$ & $1.2(0.7-1.9)-0.81$ \\
\hline Diabetes mellitus & $4.2(2.1-9.1)-0.03$ & $6.1(1.1-23.8)-0.04$ \\
\hline Coronary artery disease & $1.2(0.8-1.3)-0.61$ & $1.3(0.4-13.3)-0.69$ \\
\hline Congestive heart failure & $1.6(1.1-2.1)-0.39$ & $1.9(0.3-15.5)-0.55$ \\
\hline Cerebrovascular disease & $1.6(1.1-1.9)-0.43$ & $1.7(0.5-19.9)-0.59$ \\
\hline Smoking history & $1.2(0.8-1.9)-0.55$ & $1.3(0.3-15.3)-0.71$ \\
\hline Current smoker & $1.5(1.1-2.0)-0.45$ & $1.6(0.6-13.9)-0.61$ \\
\hline Hypertension & $1.9(1.3-3.7)-0.31$ & $2.1(0.3-21-1)-0.37$ \\
\hline Hyperlipidaemia & $1.6(1.0-1.8)-0.44$ & $1.7(0.5-17.4)-0.63$ \\
\hline Chronic renal insufficiency(eGFR $<90$ mL/min) & $3.9(1.7-8.8)-0.04$ & $4.6(0.7-18.2)-0.04$ \\
\hline \multicolumn{3}{|l|}{ Rutherford categories at presentation } \\
\hline 1 or 2 or 3 - claudication & $0.9(0.7-1.3)-0.80$ & $1.8(0.6-13.0)-0.83$ \\
\hline 4 - rest pain & $1.2(0.8-1.3)-0.62$ & $2.1(0.5-13.8)-0.71$ \\
\hline 5 or 6 - tissue loss & $4.1(1.9-8.7)-0.04$ & $2.4(0.7-10.8)-0.46$ \\
\hline Bypass graft target(Below-knee popliteal artery) & $4.3(2.2-9.3)-0.03$ & $4.1(1.2-19.2)-0.04$ \\
\hline Lesion length $\geq 30 \mathrm{~cm}$ & $4.5(2.9-9.7)-0.02$ & $4.6(0.9-14.2)-0.04$ \\
\hline TASCII D Lesion & $4.2(2.1-8.9)-0.03$ & $5.7(1.2-19.8)-0.04$ \\
\hline BTK runoff (1 vessel) & $5.5(3.1-11.1)-0.01$ & $6.8(1.1-23.9)-0.02$ \\
\hline Stents positioned per patient $\geq 3$ & $3.9(1.2-14.9)-0.04$ & $1.5(0.6-11.0)-0.48$ \\
\hline
\end{tabular}

and BTK runoff (1 vessel) (HR 6.8; $P=0.02$ ). Age, age $\geq$ 70 years, sex (male), bmi, coronary artery disease, congestive heart failure, cerebrovascular disease, smoking history, current smoker, hypertension, hyperlipidaemia, claudication/rest pain/tissue loss at clinical presentation and stents positioned per patient $\geq 3$ were not predictors of primary patency loss.

\section{Discussion}

Bypass grafting is the treatment of choice for chronic total occlusions (CTO) of the superficial femoral artery (SFA), with a favourable technical success rate and clinical prognosis (Norgren et al. 2007). However, the following occlusion of the femoropopliteal arterial bypass in patients with CLI remains a significant problem, that hardly challenges vascular surgeons and interventional radiologists due to an attendant increased mortality and morbidity (Betz et al. 2012; Greenberg and Ouriel 1998). The ideal treatment for failed femoropopliteal bypass in patients with CLI and CTOs of the native SFA would minimize postoperative morbidity and achieve satisfactory limb salvage and long-term patency rates ( $\mathrm{Li}$ et al. 2018). After a bypass graft failure has occurred, the possible targets of intervention are the bypass graft itself or the native SFA. Among bypass graft interventions, surgical or endovascular approaches can be distinguished. Performing a secondary femoropopliteal bypass is still considered the standard of care, although it is associated with a higher complication rate (perioperative morbidity of $25 \%$ ), $66 \%$ limb salvage rate at 5 years and lower mid-term patency rate in comparison with primary bypass (Baldwin et al. 2004; Belkin et al. 1995). Taha et al. reported a significantly higher overall mortality rate at 30 days and 1 year in the patients requiring open reintervention (Taha et al. 2015). Besides, advanced age, lack of a good great saphenous vein, anastomosis' pseudoaneurysms and high surgical risks make surgical approach not always suitable. An alternative surgical strategy is to perform open thrombectomy and surgical revision of the bypass graft, with or without a patch. Despite an acceptable technical success rate, this technique correlates with poor primary patency and limb salvage rates: 40 and $45 \%$, respectively, at 30 months, according to Quinones-Baldrich et al. (Quinones-Baldrich et al. 1991). Options to achieve endovascular recanalization of bypass graft include mechanical thrombectomy and catheter-directed thrombolysis (CDT). CDT, available for patients presenting with acute limb ischemia (ALI), provides a high technical success 
rate, around $84 \%$ (Gardiner et al. 1989). Nevertheless, the median patency is 8 months, the patency rate at 5 years is $19-28 \%$ and the limb salvage rate at 5 years is $55 \%$ (Nehler et al. 2003; Nackman et al. 1997). To these data are added the not negligible complications, especially hemorrhagic, and the frequent need to add a PTA treatment, after the CDT has been performed. For the aforementioned reasons, clinical outcome data about CDT remains inconsistent. More recently mechanical thrombectomy devices have been utilized alone or in combination with thrombolytics to achieve more rapid lysis (Shammas et al. 2008). Mechanical thrombectomy has been highly effective in fresh thrombus but much less effective in older, more organized thrombus, with a primary patency rate of $50 \%$ at 12 months (Domínguez Paillacho et al. 2018; Kalinowski and Waqner 2003). Hemolysis, residual thrombus, and distal embolization are recognized complications of isolated mechanical lysis.

After a femoropopliteal bypass graft failure has occurred, as an alternative to bypass itself, the other possible target of intervention is the native SFA. Over the past few years, angioplasty has been commonly used to treat chronic total occlusions of the native SFA, with a good technical success rate and clinical prognosis (Mewissen 2004). Besides, contemporary vascular literature (Baril et al. 2010; Laganà et al. 2011; Dosluoglu et al. 2008) on primary endovascular treatment of TASC II $\mathrm{C}$ and D femoropopliteal lesions has shown acceptable patency rates compared with those recorded after femoropopliteal bypass, also thanks to the development of endovascular techniques (such as subintimal angioplasty or SAFARI technique) and tools (re-entry devices, stents, etc.) (Hua et al. 2013). Hence, such a scientific background, characterized by the scarcity of alternative endovascular techniques (CDT, mechanical thrombectomy) that are effective and by the presence of still partial but interesting data concerning the patency of TASC II C and $\mathrm{D}$ lesions treated with endovascular recanalization as first-therapy, explains the rationale for considering endovascular recanalization of native SFA CTOs as a treatment hypothesis after femoropopliteal bypass failure, in patients with CLI who refuse surgery or are considered unfit for surgery.

In this study, we report the results of a retrospective analysis of data, regarding patients with CLI after the failure of femoropopliteal bypass who had undergone endovascular recanalization of CTOs of the native SFA. There was a high technical success rate $(94.4 \%)$. The experience developed with advanced endovascular techniques (such as subintimal angioplasty or SAFARI technique) and tools (re-entry devices) has certainly contributed to the results achieved, enabling the treatment of more challenging arterial lesions. Many secondary endovascular procedures for acute thrombosis have been performed, so that the primary patency rate was low at both 1 year and 5 years (61 and $46 \%$, respectively) but the secondary patency rate was considerable, at both 1 year and 5 years (93 and $61 \%$, respectively). In some cases, some authors have used the term "major adverse limb events" (MALE) which definition includes endovascular reinterventions in the same leg. This may explain why in literature there is high variability in reporting rates of adverse limb events (Davies and El-Sayed 2017), considering that many secondary endovascular procedures are often performed. The most interesting recorded results were the amputation-free and the limb salvage rates, which were approximately 93 and $94 \%$ at 1 year, 76 and $88 \%$ at 5 years, respectively. Thus, longterm limb salvage was achieved in the majority of patients. This figure is partly attributable to the preservation and enhancement of the arterial patrimony of the collateral branches, guaranteed by profunda femoris artery and by the endovascular recanalization of the native SFA with intraluminal approach and often sacrificed by the surgical approach. Even in the case of main vessel reocclusion, the collateral branches by SFA and profunda femoris artery could guarantee a blood supply, sufficient to maintain tissue integrity. Furthermore, distal BTK lesions can be treated at the same time, improving outflow. Patency and limb salvage rates at 7 years show interesting results too, but survivor function at the far right of a Kaplan-Meier survival curve should be interpreted cautiously since there are fewer patients - low numbers at risk - remaining in the study group and the survival estimates are not as accurate (Rich et al. 2010). Thus, it should be kept in mind that the Kaplan-Meier method's main focus is on the entire curve of mortality rather than on the traditional clinical concern with rates at fixed periodic intervals (Feinstein 1985).

The data presented above represent one of the largest and longest follow-ups to date and are comparable with those published previously in the few case-series studies found in the literature, concerning this topic. Gandini (Gandini et al. 2009) reported a technical success rate of $93.7 \%$, a secondary patency rate of $73.5 \%$ at 3 years and a limb salvage rate of $88 \%$ at 3 years. Davies (Davies and El-Sayed 2017) demonstrated in a retrospective review, comparing the outcomes of bypass redoing versus native SFA recanalization in patients with symptomatic femoropopliteal bypass occlusion, that the SFA recanalization group, treated with direct stenting, presented an amputation-free survival rate of $33 \% \pm 9 \%$ at 3 years. $\mathrm{Li}$ $\mathrm{Z}$ (Li et al. 2018) described a technical success rate of $95.6 \%$, a secondary patency rate of $61 \%$ at 3 years and a limb salvage rate of $95 \%$ at 3 years. In other case series, secondary patency rates at 1 year ranged from 44 to $96 \%$ and limb salvage rates were $65-96 \%$ at 2 years 
(Kawarada and Yokoi 2010; Yin et al. 2015; Wrigley et al. 2014).

Furthermore, the data presented in our study are comparable to the results of the primary endovascular treatment of TASC II C and D femoropopliteal lesions. Guo (Guo et al. 2015) reported a technical success rate of $95 \%$ and a secondary patency rate of $63 \%$ at 3 years after endovascular treatment of TASC II D femoropopliteal lesions. Dias-Neto and others (Dias-Neto et al. 2018; Joo et al. 2017; Veraldi et al. 2018) reported similar mean procedure duration and fluoroscopy time.

Data analysis reveals that considering the long-term outcome, endovascular recanalization of the native SFA may not be inferior to the bypass redoing too, the current standard of care. Previously published data on secondary femoropopliteal bypass report a primary patency rate of $28-57 \%$ and a limb salvage rate of 66$72.4 \%$ at 5 years, in patients with CLI (Belkin et al. 1995; Yang et al. 1991; Edwards et al. 1990). A more recent retrospective review by Davies (Davies and El-Sayed 2017) has demonstrated at 3 years an amputation-free survival rate of $56 \%$ in the bypass graft group. Besides, when the results are examined, the potential risk of selection bias cannot be ignored. Endovascular recanalization is generally offered only to patients who are unfit for surgery or who refuse a second surgical approach. It is possible, therefore, that the poor general clinical conditions of these patients affect the specific clinical outcome of the endovascular approach in a pejorative sense. Consequently, since the presence of selection bias in the study population cannot be excluded, the patency and limb salvage rates become even more interesting, when compared to those detected with a bypass redoing.

Therefore, in patients with CLI and failed femoropopliteal bypass, if the endovascular recanalization of the native SFA CTOs is a safe and considerable alternative, with high long-term limb salvage rates, to bypass redoing, even in those patients fit for surgery, remains an open question. Testing this hypothesis by performing a randomized controlled trial could be an interesting future perspective derived from this study. Although the data available to date do not allow to reach definitive conclusions, at least they suggest that the bypass redoing should no longer be considered as an automatic and obvious choice in all cases.

Due to a lack of previously published data, there is a shortage in the literature regarding the risk factors impacting the primary patency loss in patients who underwent endovascular recanalization of the native SFA CTOs after femoropopliteal bypass failure. By univariate analysis, statistically significant predictors of primary patency loss were diabetes mellitus, chronic renal insufficiency $(e G F R<90 \mathrm{~mL} / \mathrm{min})$, tissue loss at clinical presentation, bypass target to the below-knee popliteal artery, lesion length $\geq 30 \mathrm{~cm}$, TASC II D lesion, BTK runoff ( 1 vessel) and stents positioned per patient $\geq 3$. By multivariate analysis, statistically significant predictors of primary patency loss were diabetes mellitus, chronic renal insufficiency (eGFR $<90 \mathrm{~mL} / \mathrm{min}$ ), bypass target to the below-knee popliteal artery, lesion length $\geq 30 \mathrm{~cm}$, TASC II D lesion and BTK runoff (1 vessel). The contemporary literature on the endovascular treatment of peripheral artery disease (PAD) demonstrates that diabetes is one of the strongest risk factors for primary patency loss (DeRubertis et al. 2007). Our study has found a similar result both in the univariate $(\mathrm{HR}=4.2 ; \mathrm{CI}=$ 2.1-9.1; $P=0.03)$ and in the multivariate $(\mathrm{HR}=6.1 ; \mathrm{CI}=$ $1.1-23.8 ; P=0.04$ ) analysis. Hence, greater consideration should be given when native lesions are endovascularly treated in diabetic patients since they were more likely to have accelerated disease progression over time due to their diabetic status (Bakken et al. 2007). Chronic renal insufficiency (eGFR $<90 \mathrm{~mL} / \mathrm{min}$ ) independently predicts primary patency loss, due to its known capacity to cause a more advanced and rapidly progressive arterial occlusive disease (Iida et al. 2014). In agreement with previously published data by Scali ST (Scali et al. 2011), a BTK runoff of one vessel is a strong statistically significant predictor of primary patency loss, perhaps also a surrogate of the overall duration, severity and limbextension of the atherosclerotic disease. Another important finding is that a lesion length $\geq 30 \mathrm{~cm}$ and a TASC II $\mathrm{D}$ lesion are predictors of primary patency loss. This finding is in contrast with one of the few previously published reports on the endovascular recanalization of the native SFA CTOs in patients with CLI after failed femoropopliteal bypass ( $\mathrm{Li}$ et al. 2018), whose regression analysis had not shown significant influence of a lesion length $>20 \mathrm{~cm}$ and a TASC II D lesion on the primary patency loss, but it's in agreement with the historical and contemporary vascular literature on primary endovascular therapy for lower limb lesions (Jørgensen et al. 1991; Iida et al. 2014). In particular, 2017 ESC guidelines on the diagnosis and treatment of PAD (Aboyans et al. 2018) clearly state that if the occlusion/stenosis is > $25 \mathrm{~cm}$, endovascular recanalization is still possible, but better long-term patency is achieved with surgical bypass, especially when using the great saphenous vein, although it does not distinguish between primary patency and secondary patency rates.

The below-knee popliteal artery bypass target heralds the presence of a more extensive TASC II D disease of the native SFA, strongly associated with a higher rate of restenosis after a percutaneous intervention (Owens et al. 2008a, b). It's well-known that patients with tissue loss at clinical presentation have poorer outcomes (Setacci et al. 2009), not only due to atherosclerotic disease extended to BTK-district but also because of poorer 
blood supply by collateral circulation at the femoropopliteal district, in turn, a sign of a severe and extended disease of the native SFA. The last predictor of primary patency loss is a number $\geq 3$ of stents positioned per patient. The lack of significance according to the multivariate analysis and the wide $\mathrm{CI}$ generated by the univariate analysis $(\mathrm{HR}=3.9 ; \mathrm{CI}=1.2-14.9 ; P=0.04)$ might suggest the imprecision of the univariate estimate. Hence, a number $\geq 3$ of stents positioned per patient might be just a surrogate for other risk factors independently associated with a poorer outcome and a PTA failure, such as a TASC II D lesion, a more extensive or calcified disease. In addition, there is growing evidence on the effectiveness of the full metal jacket approach in the treatment of TASC II C and D lesions (Laganà et al. 2011; Philips et al. 2018).

Limitations of the study are the lack of a control group, the single-centre setting, the retrospectivity of the analysis and the scarcity of data in the literature, necessary to evaluate the congruence and the consistency of the data presented.

\section{Conclusions}

The endovascular recanalization of chronic total occlusions (CTO) of the native superficial femoral artery (SFA) after failed femoropopliteal bypass is a safe and effective therapeutic option in patients unfit for surgery with critical limb ischemia. Besides, the results presented represent one of the largest and longest follow-ups ever published and contribute to the small body of literature present on this topic to date.

\section{Abbreviations}

ABI: Ankle-brachial index; ALI: Acute limb ischemia; BTK: Below-the-knee; CDT: Catheter-directed thrombolysis; Cl: Confidence interval; CLI: Critical limb ischemia; CT: Computed tomography; CTO: Chronic total occlusions; GFR: Glomerular filtration rate; GSV: Great saphenous vein; HR: Hazard ratio; IQR: Interquartile range; MALE: Major adverse limb events; PAD: Peripheral artery disease; PTFE: Polytetrafluoroethylene; SFA: Superficial femoral artery; TASC II: TransAtlantic Inter-Society Consensus for the Management of Peripheral Arterial Disease II
}

\section{Acknowledgements}

Not applicable.

\section{Authors' contributions}

RM and DL conceived the study concepts and design. RM, MT, MA and SC performed literature research and assisted in data collection. RM, ML and GG were in charge of manuscript editing. MM performed the statistical analysis using the provided database. All authors read and approved the final manuscript.

\section{Funding}

Not applicable.

\section{Availability of data and materials}

The datasets used and/or analysed during the current study are available from the corresponding author on reasonable request.

\section{Declarations}

Ethics approval and consent to participate

Approval by the ethic committee of Magna Graecia University and written informed consent by patients were obtained.

\section{Consent for publication}

Not applicable.

\section{Competing interests}

The authors declare that they have no competing interests.

\section{Author details}

${ }^{1}$ Radiology Division, Department of Experimental and Clinical Medicine, Magna Graecia University of Catanzaro, University Hospital Mater Domini, Viale Europa, 88100 Catanzaro, Italy. ${ }^{2}$ Digestive Surgery Unit, Science of Health Department, Magna Graecia University, Catanzaro, Italy. ${ }^{3}$ Cardiology Division, Giovanni Paolo II Hospital, Lamezia Terme, Italy. ${ }^{4}$ Cardiovascular Disease Unit, San Giovanni di Dio Hospital, Crotone, Italy. ${ }^{5}$ nstitute for high performance computing and networking (ICAR), National Research Council (Cnr), Rende, Italy. ${ }^{6}$ Vascular Surgery Division, University Hospital Mater Domini, Catanzaro, Italy. ${ }^{7}$ Radiology Division, Azienda

Ospedaliero-Universitaria "Maggiore della Carità", Novara, Italy.

Received: 10 May 2021 Accepted: 23 August 2021

Published online: 07 September 2021

\section{References}

Ahn SS, Rutherford RB, Becker GJ et al (1993) Reporting standards for lower extremity arterial endovascular procedures. Society for Vascular Surgery/ International Society for Cardiovascular Surgery. J Vasc Surg 17:1103-1107

Bakken AM, Palchik E, Rhodes JM, Saad WE, Davies MG (2007) Impact of diabetes mellitus on outcomes of superficial femoral artery endoluminal interventions. J Vasc Surg 46:946-958

Baldwin ZK, Pearce BJ, Curi MA et al (2004) Limb salvage after infrainguinal bypass graft failure. J Vasc Surg 39:951-957

Baril DT, Chaer RA, Rhee RY, Makaroun MS, Marone LK (2010) Endovascular interventions for TASC II D femoropopliteal lesions. J Vasc Surg 51:1406-1412

Belkin M, Conte MS, Donaldson MC, Mannick JA, Whittemore AD (1995) Preferred strategies for secondary infrainguinal bypass: lessons learned from 300 consecutive reoperations. J Vasc Surg 21:282-293

Betz T, Uhl C, Steinbauer M et al (2012) Operative or interventional treatment in infrainguinal bypass occlusion: are there predictive factors affecting outcome? Surg Sci 3:473-478

Brewster DC, LaSalle AJ, Robison JG, Strayhorn EC, Darling RC (1983) Femoropopliteal graft failures. Clinical consequences and success of secondary reconstructions. Arch Surg 118:1043-1047

Davies MG, El-Sayed HF (2017) Outcomes of native superficial femoral artery chronic total occlusion recanalization after failed femoropopliteal bypass. J Vasc Surg 65(3):726-733

DeRubertis BG, Faries PL, McKinsey JF et al (2007) Shifting paradigms in the treatment of lower extremity vascular disease: a report of 1000 percutaneous interventions. Ann Surg 246:415-422

Dias-Neto M, Matschuck M, Bausback Y, Banning-Eichenseher U, Steiner S, Branzan D, Staab H, Varcoe RL, Scheinert D, Schmidt A (2018) Endovascular treatment of severely calcified femoropopliteal lesions using the "pave-andcrack" technique: Technical description and 12-month results. J Endovasc Ther 25(3):334-342. https://doi.org/10.1177/1526602818763352

Donaldson MC, Mannick JA, Whittemore AD (1991) Femoral-distal bypass with in situ greater saphenous vein. Long-term results using the Mills valvulotome. Ann Surg 213:457-464

Dosluoglu HH, Cherr GS, Lall P, Harris LM, Dryjski ML (2008) Stenting vs above knee polytetrafluoroethylene bypass for TransAtlantic InterSociety Consensus॥ C and D superficial femoral artery disease. J Vasc Surg 48:1166-1174

Edwards JE, Taylor LM, Porter JM (1990) Treatment of failed lower extremity bypass grafts with new autogenous vein bypass grafting. J Vasc Surg 11:136144

Feinstein AR (1985) The architecture of clinical research. In: Co (ed) Clinical Epidemiology. WB Saunders, Philadelphia

Gandini R, Chiappa R, Di PM et al (2009) Recanalization of the native artery in patients with bypass failure. Cardiovasc Intervent Radiol 32:1146-1153 
Gardiner GA Jr, Harrington DP, Koltun W, Whittemore A, Mannick JA, Levin DC (1989) Salvage of occluded arterial bypass grafts by means of thrombolysis. J Vasc Surg 9:426-431

Greenberg RK, Ouriel K (1998) A multi-modal approach to the management of bypass graft failure. Vasc Med 3:215-220

Guo X et al (2015) Outcomes of endovascular treatment for patients with TASC II D femoropopliteal occlusive disease: a single center study. BMC Cardiovasc Dis 15:44

Hua WR et al (2013) Popliteal versus tibial retrograde access for subintimal arterial flossing with antegrade-retrograde intervention (SAFARI) technique. Eur J Vasc Endovasc Surg 46(2):249-254

lida O, Takahara M et al (2014) Shared and differential factors influencing restenosis following endovascular therapy between TASC (Trans-Atlantic Inter-Society Consensus) II class A to C and D lesions in the femoropopliteal artery. JACC 7:792-798

Joo HJ, Jang DH, Yu CW, Choi YJ, Park J, Lee HJ, Park JH, Hong SJ, Lim DS (2017) Efficacy and safety of endovascular treatment for femoropopliteal lesions of TASC II type C and D compared with TASC II type A and B in Korea. Vascular 25(4):351-358. https://doi.org/10.1177/1708538116678539

Jørgensen B, Tønnesen KH, Holstein P (1991) Late hemodynamic failure following percutaneous transluminal angioplasty for long and multifocal femoropopliteal stenoses. Cardiovasc Intervent Radiol 14:290-292

Kalinowski M, Waqner HJ (2003) Adjunctive techniques in percutaneous mechanical thrombectomy. Tech Vasc Interv Radiol 6(1):6-13

Kawarada O, Yokoi Y (2010) Native chronic total occlusion recanalization after lower limb bypass graft occlusion: a series of nine cases. Catheter Cardiovasc Interv 76:214-219

Laganà D, Carrafiello G, Barresi M, Lumia D, Dizonno M, Vizzari FA et al (2011) "Full metal jacket" with direct stenting of complete chronic occlusions of the superficial femoral artery. Radiol Med 116:444-453

Leather RP, Shah DM, Chang BB, Kaufman JL (1988) Resurrection of the in situ saphenous vein bypass. 1000 cases later. Ann Surg 208:435-442

Li Z, Feng R, Qin F, Zhao Z et al (2018) Recanalization of native superficial femoral artery chronic total occlusion after failed femoropopliteal bypass in patients with critical limb ischemia. J Interv Cardiol 31(2):207-215

Mewissen MW (2004) Self-expanding nitinol stents in the femoropopliteal segment: technique and mid-term results. Tech Vasc Interv Radiol 7:2-5

Nackman GB, Walsh DB, Fillinger MF et al (1997) Thrombolysis of occluded infrainguinal vein grafts: predictors of outcome. J Vasc Surg 25:1023-1031

Nehler MR, Mueller RJ, McLafferty RB et al (2003) Outcome of catheter-directed thrombolysis for lower extremity arterial bypass occlusion. J Vasc Surg 37:72-78

Owens CD, Ho KJ, Conte MS (2008a) Lower extremity vein graft failure: a translational approach. Vasc Med 13:63-74

Owens CD, Ho KJ, Conte MS (2008b) Risk factors for failure of lower-extremity revascularization procedures: are they different for bypass and percutaneous procedures? Semin Vasc Surg 21:143-153

Phillips JA, Falls A, Kolluri R, Whipp A, Collins C, Mohir-Sadaai S, Reid B, Patil N, Alston M, Troyan M, Ansel GM (2018) Full drug-eluting stent jacket: two-year results of a single-center experience with zilver PTX stenting for long lesions in the femoropopliteal arteries. J Endovasc Ther 25(3):295-301. https://doi. org/10.1177/1526602818762805

Quiñones-Baldrich WJ, Prego A, Ucelay-Gomez R, Vescera CL, MooreWS (1991) Failure of PTFE infrainguinal revascularization: patterns, management alternatives, and outcome. Ann Vasc Surg 5:163-169

Rich JT, Neely JG et al (2010) A practical quide to understanding Kaplan-Meier curves. Otolaryngol Head Neck Surg 143(3):331-336

Rutherford RB, Baker JD, Ernst C et al (1997) Recommended standards for reports dealing with lower extremity ischemia: revised version. J Vasc Surg 26:517-538

Scali ST, Rzucidlo EM, Bjerke AA et al (2011) Long-term results of open and endovascular revascularization of superficial femoral artery occlusive disease. J Vasc Surg 54:714-721

Setacci C, Chisci E, de Donato G, Setacci F, lacoponi F, Galzerano G,et al (2009) Subintimal angioplasty with the aid of a re-entry device for TASCC and D lesions of the SFA. Eur J Vasc Endovasc Surg 38:76-87

Shammas NW, Dippel EJ, Shammas G, Gayton L, Coiner D, Jerin M (2008) Dethrombosis of lower extremity arteries using the power-pulse spray technique in patients with recent onset thrombotic occlusions: results of the DETHROMBOSIS registry. J Endovasc Ther 15(5):570-579

Taha AG, Byrne RM et al (2015) Comparative effectiveness of endovascular versus surgical revascularization for acute lower extremity ischemia. J Vasc Surg 61 : 147-154
Taylor LM, Edwards JM, Porter JM (1990) Present status of reversed vein bypass grafting: five-year results of a modern series. J Vasc Surg 11:193-205

Veraldi GF, Mezzetto L, Macrì M, Criscenti P, Corvasce A, Poli R (2018) Comparison of endovascular versus bypass surgery in femoropopliteal TASC II D lesions: a single-center study. Ann Vasc Surg 47:179-187. https://doi.org/10.1016/j.a vsg.2017.09.008

Wrigley CW, Vance A, Niesen T et al (2014) Endovascular recanalization of native chronic total occlusions in patients with failed lower-extremity bypass grafts. J Vasc Interv Radiol 25:1353-1359

Yang PM, Wengerter KR, Veith FJ, Panetta TF, Nwosisi C, Gupta SK (1991) Value and limitations of secondary femoropopliteal bypasses with polytetrafluoroethylene. J Vasc Surg 14:292-298

Yin M, Wang W, Huang X et al (2015) Endovascular recanalization of chronically occluded native arteries after failed bypass surgery in patients with critical ischemia. Cardiovasc Intervent Radiol 38:1468-1476

Ziegler KR, Muto A, Eghbalieh SD, Dardik A (2011) Basic data related to surgical infrainguinal revascularization procedures: a twenty year update. Ann Vasc Surg 25:413-422

Norgren L, Hiatt WR, Dormandy JA et al (2007) Inter-society consensus for the management of peripheral arterial disease (TASC II). J Vasc Surg 45 Suppl S: S5-67

Domínguez Paillacho ID et al (2018) Mechanical thrombectomy with ANGIOJET system in patients with lower limb Bypass occlusion. ECR 2018\&nbsp;C-1713

Aboyans V, Ricco JB, Bartelink MEL, Björck M, Brodmann M, Cohnert T, Collet JP, Czerny M, De Carlo M, Debus S, Espinola-Klein C, Kahan T, Kownator S, Mazzolai L, Naylor AR, Roffi M, Röther J, Sprynger M, Tendera M, Tepe G, Venermo M, Vlachopoulos C, Desormais I; ESC Scientific Document Group (2018) 2017 ESC Guidelines on the Diagnosis and Treatment of Peripheral Arterial Diseases, in collaboration with the European Society for Vascular Surgery (ESVS): Document covering atherosclerotic disease of extracranial carotid and vertebral, mesenteric, renal, upper and lower extremity arteries Endorsed by: the European Stroke Organization (ESO) The Task Force for the Diagnosis and Treatment of Peripheral Arterial Diseases of the European Society of Cardiology (ESC) and of the European Society for Vascular Surgery (ESVS). Eur Heart J 39(9):763-816. https://doi.org/10.1093/eurheartj/ehx095

\section{Publisher's Note}

Springer Nature remains neutral with regard to jurisdictional claims in published maps and institutional affiliations.

\section{Submit your manuscript to a SpringerOpen ${ }^{\circ}$ journal and benefit from:}

- Convenient online submission

- Rigorous peer review

- Open access: articles freely available online

High visibility within the field

- Retaining the copyright to your article

Submit your next manuscript at $\boldsymbol{\sim}$ springeropen.com 